\title{
Fuzzy Logic based Model to Reprioritize Cloud Computing Process Requests using Extended Parameters
}

\author{
Pooja Chopra \\ Research Scholar \\ Department of Computer Applications \\ I.K.G PTU, Jalandhar, India
}

\author{
R. P. S. Bedi,PhD \\ Controller of Examinations \\ I.K.G PTU, Jalandhar, India
}

\begin{abstract}
In the recent years, there is a wide increase in the demand of cloud computing because of its endless advantages like reduced infrastructure cost, scalability, virtualization, on demand service etc. This technology has brought a great revolution in the field of Information Technology.

Resource Provisioning is an area in cloud computing where resources are provisioned to the processes in such a way that every coming process can get its demanded resource in time and can complete its execution in time and that too with full privacy. For our model proposed, we have taken existing work in hybrid cloud environment. We have used Fuzzy Logic as a tool for redefining the priorities to the processes. In this paper, a Fuzzy Logic based model is proposed to reprioritize Cloud Computing process requests using extended parameters. The central idea is to develop a conceptual model for prioritizing processes on the basis of their age, execution time and security factors. For considering these factors, human expertise is needed. Therefore, we have incorporated Fuzzy Logic in the system where the Fuzzy inference system will decide the priorities of the processes.
\end{abstract}

\section{Keywords}

Cloud Computing, Hybrid Cloud, Resource Provisioning, Fuzzy logic, Fuzzy Inference System.

\section{INTRODUCTION}

Cloud Computing is a new innovation that give a new way to success in Information technology. Cloud Computing is the use of Cloud resources (hardware and software) that is delivered as a service over a network [1].Concepts like ondemand self service, broad network access, resource pooling and other trademarks of cloud computing services are the key components of its current popularity [2].Actually, it is a internet based technology where the resources are provided on demand like storage, networking tools, servers, software applications and also platform on which customers can build and deploy their applications. These resources are provided as services to the customers. The customers don't know where these resources are. They only use these resources and pay on the basis of resources demanded.

\subsection{Types of services}

\subsubsection{Software as a Service (SAAS):}

SAAS is a software delivery model in which applications are accessed by a simple interface such as a web browser over the internet [3]. The user needs not to spend on the costly softwares. They use these softwares through cloud and pay on the basis of usage

\subsubsection{Platform as a Service (PAAS):}

PAAS provide a computing platform using the cloud infrastructure [4] where the user can build and deploy their applications

\subsubsection{Infrastructure as a service (IAAS):}

IAAS provides the consumer the processing, storage, networks and other fundamental computing resources where the consumer is able to deploy and run arbitrary software which can include operating systems and applications [5]

\subsection{TYPES OF CLOUDS}

1.2.1 Private Cloud: Private Cloud is a distinct and secure environment, only the specified client can operate [6].It is used by the organization which owns it.

1.2.2 Public Cloud: Any one on the internet can access public cloud.

1.2.3. Hybrid Cloud: Intermixing of public cloud and private cloud is hybrid cloud. The features of public cloud (Low cost and scalability) are not there in private cloud and features of private cloud (Security and Privacy) are not there in public cloud. So in order to enjoy the benefits of both clouds, one can use hybrid cloud.

1.2.4Community Cloud: It is a model in which cloud services are shared by multiple organizations and supports a specific community that has special concerns(e.g. mission, security considerations etc.It may be managed by the organizations itself or some third party, either onpremise or off-premise[7]

\subsection{Resource Provisioning}

Resource provisioning helps in determining that which and how much resources are required for the submitted workloads so that QOS parameters such as security, availability, reliability and CPU utilization can be maintained [8]. In fact, determining right amount of resources need to be provisioned is a very complex task. The amount of resources should be minimum for a workload to maintain a desirable level of service quality or maximize throughput (or minimize workload completion time) of a workload [9].Various cloud based resource provisioning mechanisms are there in the existing literature which have explained in our review paper [8] are explained briefly in the next section. 


\subsection{Resource Provisioning Mechanisms}

Various cloud based resource provisioning mechanisms are as follows:

- HYBRID CLOUD BASED: In this category, resource provisioning schemes has been proposed where researcher has taken more than one cloud to improve scalability. Resources have been allocated to the processes on the basis of priority of the process. High Priority processes go to private cloud for resources whereas medium and low priority processes go to public cloud for resources. Proposed approaches proved to be cost-effective while increasing the resource utilization.[10][11][12].

- Reliability Based: This policy takes care of resource provisioning in cloud based environment while improving reliability of the virtual machines providing these resources[13].Various brokering strategies have been proposed while modifying the backfilling scheduling algorithm to give a fault free environment for private cloud for provisioning resources.[14]

- Queuing Model Based: A dynamic resource provisioning mechanism is proposed while removing deadlocks among the processes requesting resources [15].

- Ontology Based: An Inter Cloud Resource Provisioning Scheme is proposed and the researcher addressed the problem of interoperability between the clouds with the help of ontology [16].

- Deadline Based: The researcher proposed deadline driven resource provisioning algorithm for cloud application platform ANEKA while reducing application execution time [17].

- Service Level agreement (SLA) based: Resource provisioning policy for heterogeneous clouds is proposed by considering their SLA.The policy results in maximum utilization of resources also by decreasing risk of underutilization of resources [18].

- Cost Based: A cost effective resource provisioning policy is proposed adjusting in multiple private and public clouds[19]

- Application Based: Cloud based brokering strategy is proposed where the resources are provisioned from the best suited service provider and results in decreasing cost and promotes scalability and robustness [20].

\section{MOTIVATION}

We have taken the base policy presented by the researcher [10] in which resources are provisioned in hybrid cloud based environment. Priorities have been assigned to the coming processes. High priority processes always go to private cloud and Low priority and medium priority processes go to public cloud or private cloud depending upon availability of cloud. But the approach is totally uni-dimensional for allocating priorities to the processes. Moreover, there is no solution to the problem of priority conflict where processes with same priorities arrive.

\subsection{Why Fuzzy Logic?}

We have used Fuzzy Logic tool in the proposed technique to solve the above given issues. Fuzzy Logic is a branch of Artificial Intelligence that has been proposed by Zadeh in 1965.Fuzzy Logic provides a simple way to arrive at a definite conclusion based upon vague, ambiguous, imprecise, noisy or missing input information [21].

Fuzzy Logic is more than simple true or False that is supported by conventional systems. Fuzzy Logic is a collection of membership functions and rules that support multi-valued logic as the degree of truthfulness varies between true and false.

Due to excellent response of fuzzy Logic to uncertainty and its logically reasoning, Fuzzy Logic is widely applied in Cloud Computing [22].

\subsection{Applications of Fuzzy Logic in Cloud Computing}

On the basis of a literature reviewed in our previous paper [23] various applications of Fuzzy Logic in the field of Cloud Computing have been listed as follows:

- Load Balancing[24][25]

- Job Scheduling [26][27][28]

- Trust Evaluation[29]

- Power monitoring[30]

- QOS optimization [31].

- Improving Reliability [32] [33].

\subsection{Application of Fuzzy Logic In Proposed Model}

Fuzzy Logic has proved beneficial in various real life applications as it works like human mind. That's why we are also using Fuzzy Logic to improve the existing model.

We have used Fuzzy Logic to deal with coming Cloud Computing based processes for the resources. We have designed a Fuzzy Inference System (FIS) that will allocate priorities to the processes on the basis of three factors: Security, Age and Execution Time. So we are designing three dimensional model for reprioritizing the processes in cloud computing. This also reduces the priority conflict situation to great extent.

\section{PROPOSED MODEL}

The design of Fuzzy Logic based model for giving priorities to cloud computing based processes is given in this section. Infact, Fuzzy logic is a four step process that is summarized as follows:

- Fuzzification: Fuzzification defines the membership functions for the linguistic variables. While variables in mathematics usually take numerical values, in fuzzy Logic applications, the non-numeric linguistic variables are often used to facilitate the expression of rules and facts[34].For instance, we defined age into five linguistic terms: Very Low, Low, Medium, High and Very High. Details of membership functions for input and output variables in our research is shown later.

- Rule Base: If-Then Rules are used to design rule base. For instance we have defined a rule 
If (Security based priority is High) and (age is Very Low) and (Execution Time is Low) then (allocated Priority is High)

Usually, for each linguistic term, a corresponding fuzzy set can either be designed with the help of expert(s) or derived from existing data to meet the definition of a fuzzy set [35].

- Application of Rules: On the basis of various inputs, various rules with their strengths are fired that help to arrive at definite conclusion for defining appropriate priority to a process.

- Defuzzification: The defuzzification process converts the fuzzy output into a crisp value. In our research, we have taken Allocated Priority as output variable that will define a crisp result in terms of priority allocated to the Cloud Computing process.

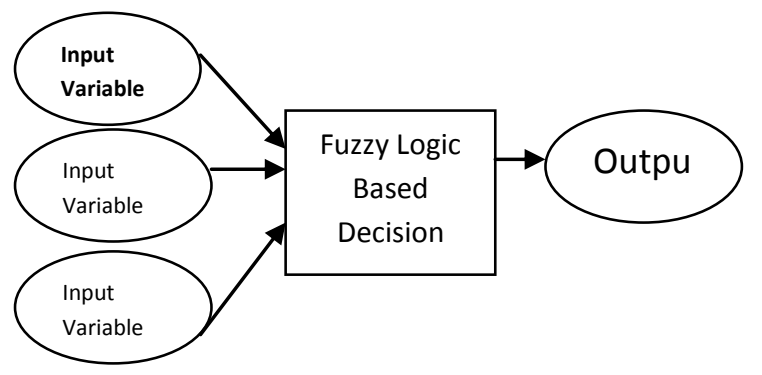

Fig 1: Fuzzy Logic Based Expert System

A Fuzzy Logic system deals with the statements that are not well defined.

For instance using conventional systems the age of a process is defined as high or low but question is up to what extent it is high? High is an imprecise term. So membership functions define the degree of something.

\subsection{Designing membership functions for input variables and output variable}

Three input variables and one output variable along with the membership functions have been defined which are given below.

\subsubsection{Input variables1:Security}

Security means how much security factor of a process is to be considered. For security, we have taken triangular membership function. For sharp transitions, we use triangular functions

$\mu_{\mathrm{L}}(\mathrm{x})=\left\{\begin{array}{c}0 \text { if } \mathrm{x} \leq .02684 \\ (\mathrm{X}-.02684) /(.2765-.02684) \\ \text { If } \mathrm{x} \in(.02684, .2765) \\ (.5261-\mathrm{x}) /(.5261-.2765) \\ \text { If } \mathrm{x} \in(.2765, .5261) \\ 0 \text { if } \mathrm{x} \geq .5261\end{array}\right.$

\subsubsection{Input variables2: Age}

For smooth transitions, we use Gaussian membership functions. So we use this type of function in age.

$\left.\begin{array}{l}\mu_{\mathrm{L}}(\mathrm{x})=\exp \left[-(\mathrm{x}-.25)^{2} / 2 * .1062^{2}\right. \\ \mu_{\mathrm{H}}(\mathrm{x})=\exp \left\{-(\mathrm{x}-.75)^{2} / 2 * .1062^{2}\right.\end{array}\right]$

\subsubsection{Input variables3: Execution Time}

Here we have used triangular Function.

$\mu_{\mathrm{M}}(\mathrm{x})=\left\{\begin{array}{c}0 \text { if } \mathrm{x} \leq .2499 \\ (\mathrm{x}-.2499) /(.5-.2499) \\ \text { If } \mathrm{x} \in(.2499, .5) \\ (.7501-\mathrm{x}) /(.7501-.5) \\ \text { If } \quad \mathrm{x} \in(.5, .7501) \\ 0 \quad \text { if } \mathrm{x} \geq .7501\end{array}\right.$

\subsubsection{Output variable: Allocated Priority}

Here we have used triangular function.

$\mu_{\mathrm{H}}(\mathrm{x})=\left\{\begin{array}{c}0 \text { if } \mathrm{x} \leq .4999 \\ (\mathrm{x}-.4999) /(.75-.4999) \\ \text { If } \mathrm{x} \in(.4999, .75) \\ (1-\mathrm{x}) /(1-.75) \\ \text { If } \mathrm{x} \in(.75,1) \\ 0 \text { if } \mathrm{x} \geq 1\end{array}\right.$

\subsection{Designing Fuzzy Rules}

The Fuzzy Logic based expert system takes inputs and on the basis of Rule Base, an output in the form of crisp value is derived. Here, we designed inputs parameters as:

Security has been defined as Very low, Low, Medium, High and Very High.

Age has been defined as Very low, Low, Medium, High and Very High.

Execution Time has been defined as Very low, Low, Medium, High and Very High.

One output will be received in the form of Allocated Priority as Very Low, Low, Medium, High and Very High.

Some of the rules of our rule base are presented below:

R1: If (Security is Very High) and (Age is Very High) and (Execution Time is Very High) Then (Allocated Priority is High)

R2: If (Security is Very High) and (Age is very High) and (Execution Time is Very Low) Then (Allocated Priority is Very High)

R10: If (Security is High) and (Age is Medium) and (Execution Time is High) Then (Allocated Priority is High) 
R40: If (Security is Very Low) and (Age is Medium) and (Execution Time is Medium) Then (Allocated Priority is Low)

Our rule base is designed with 57 rules on the basis of various experts in industries.

\subsection{Inference Mechanism}

Various commonly used Fuzzy Rules based models are:

$$
\begin{array}{ll}
1 & \text { The Mamdani } \\
2 & \text { The Takagi-Sugeno-Kang } \\
3 & \text { The Standard Additive Model }
\end{array}
$$

Here, we have taken The Mamdani method which captures expert's knowledge and is widely used in developing Decision Support Systems. Here If-Then rules format are used and Defuzzification method is used to compute the crisp output.

\subsection{Defuzzification}

As we want a single quantitative value, the output of the system is defuzzified using Centroid method.

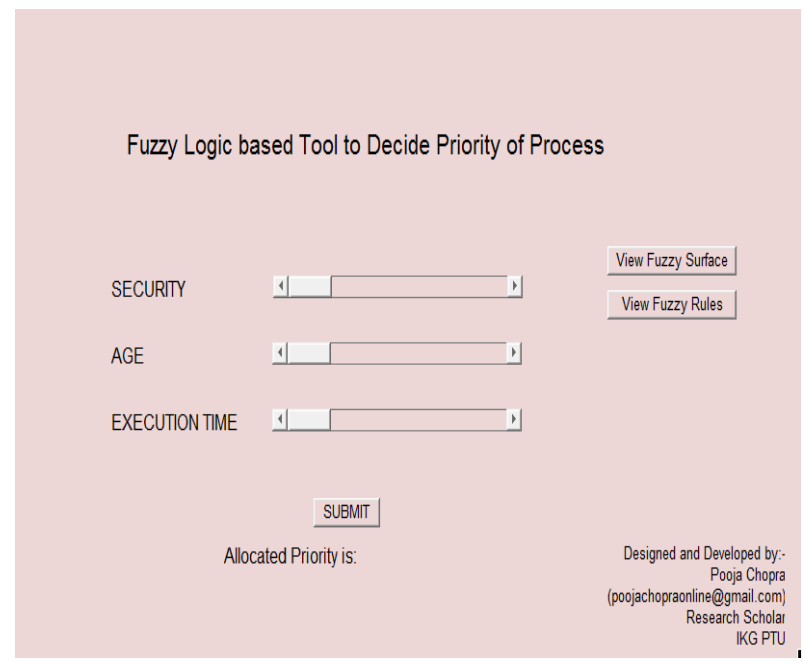

Fig 2.Graphical User Interface for the proposed system

Here the input variables i.e. Security, Age and Execution Time are taken .Submit Button is pressed and will give output in the form of Allocated Priority.

\section{FUTURE WORK}

In our future work, we will implement the above model designed where FIS will give priorities to the coming Cloud Computing based Processes.Results of both the approaches will be compared and we will show the effectiveness of fuzzy logic in the proposed technique. Also we will use another artificial intelligence technique Neural Network to perform the same work in place of Fuzzy Logic and will analyze the effect of Artificial Intelligence tools in Cloud Computing based Resource Provisioning.

\section{CONCLUSION}

A FL based model is proposed to re-prioritize the cloud computing based processes to give the order to the processes in which the processes will go to private cloud and public cloud for resources. In our model, Rule Base has been designed on the basis of Experts opinions. Accordingly FIS will determine priority of the processes in Cloud Computing environment. A simplified GUI is presented for making the system user-friendly for the users. Fuzzy Logic has proved very beneficial in real life applications. Actually Fuzzy Logic works on the mind of human being. That is the primary reason we are using Fuzzy Logic in our model.

\section{REFERENCES}

[1] Manvi, S.S. and Shyam, G.K., 2014. Resource management for Infrastructure as a Service (IaaS) in cloud computing: A survey. Journal of Network and Computer Applications, 41, pp.424-440.

[2] Endo, P.T., Rodrigues, M., Gonçalves, G.E., Kelner, J., Sadok, D.H. and Curescu, C., 2016. High availability in clouds: systematic review and research challenges. Journal of Cloud Computing, 5(1), p.16.

[3] Alam, M.I., Pandey, M. and Rautaray, S.S., 2015. A comprehensive survey on cloud computing. International Journal of Information Technology and Computer Science (IJITCS), 7(2), p.68.

[4] Jadeja, Y. and Modi, K., 2012, March. Cloud computingconcepts, architecture and challenges. In Computing, Electronics and Electrical Technologies (ICCEET), 2012 International Conference on (pp. 877-880). IEEE.

[5] Goyal, S., 2014. Public vs private vs hybrid vs community-cloud computing: a critical review. International Journal of Computer Network and Information Security, 6(3), p.20.

[6] Rao, T.V.N., Naveena, K. and David, R., 2015. A New Computing Envornment Using Hybrid Cloud. Journal of Information Sciences and Computing Technologies, 3(1), pp.180-185.

[7] Kaur, K., 2016. A Review of Cloud Computing Service Models. International Journal of Computer Applications IJCA, 140, pp.15-18.

[8] Chopra, P. and Bedi, R.P.S., STUDY OF CLOUD COMPUTING TECHNIQUES: RESOURCE PROVISIONING ASPECT.

[9] Singh, S. and Chana, I., 2016. Cloud resource provisioning: survey, status and future research directions. Knowledge and Information Systems, 49(3), pp.1005-1069.

[10] Grewal, R.K. and Pateriya, P.K., 2013. A rule-based approach for effective resource provisioning in hybrid cloud environment. New Paradigms in Internet Computing, 203, pp.41-57.

[11] Marria, N. and Pateriya, K., 2011. On-Demand Resource Provisioning In Sky Environment. International journal of Computer Science and its applications, pp.275-280.

[12] Choudhury, K., Dutta, D. and Sasmal, K., 2013. Resource Management in a Hybrid Cloud Infrastructure. International Journal of Computer Applications, 79(12)

[13] Tian, G. and Meng, D., 2010, September. Failure rules based node resource provision policy for cloud computing. In Parallel and Distributed Processing with Applications (ISPA), 2010 International Symposium on (pp. 397-404). IEEE.

[14] Javadi, B., Abawajy, J. and Buyya, R., 2012. Failureaware resource provisioning for hybrid Cloud infrastructure. Journal of parallel and distributed computing, 72(10), pp.1318-1331. 
[15] Sood, S.K., 2013. Dynamic resource provisioning in cloud based on queuing model. International Journal of Cloud Computing and Services Science, 2(4), p.313.

[16] Nelson, V. and Uma, V., 2012, April. Semantic based resource provisioning and scheduling in inter-cloud environment. In Recent Trends in Information Technology (ICRTIT), 2012 International Conference on (pp. 250-254). IEEE.

[17] Vecchiola, C., Calheiros, R.N., Karunamoorthy, D. and Buyya, R., 2012. Deadline-driven provisioning of resources for scientific applications in hybrid clouds with Aneka. Future Generation Computer Systems, 28(1), pp.58-65.

[18] Garg, S.K., Toosi, A.N., Gopalaiyengar, S.K. and Buyya, R., 2014. SLA-based virtual machine management for heterogeneous workloads in a cloud datacenter. Journal of Network and Computer Applications, 45, pp.108-120.

[19] Liu, F., Luo, B. and Niu, Y., 2017. Cost-Effective Service Provisioning for Hybrid Cloud Applications. Mobile Networks and Applications, 22(2), pp.153-160.

[20] Subramanian, T. and Savarimuthu, N., 2016. Application based brokering algorithm for optimal resource provisioning in multiple heterogeneous clouds. Vietnam Journal of Computer Science, 3(1), pp.57-70.

[21] Singh, B. and Mishra, A.K., 2015. Fuzzy Logic Control System and its Applications. International Research Journal of Engineering and Technology (IRJET), 2(8).

[22] Hayat, B., Kim, K.H. and Kim, K.I., 2017. A study on fuzzy logic based cloud computing. Cluster Computing, pp.1-15.

[23] Chopra, P. and Bedi , RPS., 2017. Applications of Fuzzy Logic in Cloud Computing: A Review. International Journal of Scientific Engineering \& Technology, pp.1083-86.

[24] Sethi, S., Sahu, A. and Jena, S.K., 2012. Efficient load balancing in cloud computing using fuzzy logic. IOSR Journal of Engineering, 2(7), pp.65-71.

[25] Susila, N., Chandramathi, S. and Kishore, R., 2014. A fuzzy-based firefly algorithm for dynamic load balancing in cloud computing environment. Journal of Emerging Technologies in Web Intelligence, 6(4), pp.435-440.
[26] Singh, I. and Arora, A., 2015. Fuzzy Based Improved Multi Queue Job Scheduling For Cloud Computing. International Journal of Advanced Research in Computer Science, 6(5)

[27] Javanmardi, S., Shojafar, M., Amendola, D., Cordeschi, N., Liu, H. and Abraham, A., 2014. Hybrid job scheduling algorithm for cloud computing environment. In Proceedings of the Fifth International Conference on Innovations in Bio-Inspired Computing and Applications IBICA 2014 (pp. 43-52). Springer, Cham.

[28] Kumar, V.V. and Dinesh, K., 2012. Job scheduling using fuzzy neural network algorithm in cloud environment. Bonfring International Journal of Man Machine Interface, 2(1), p.1.

[29] Selvaraj, A. and Sundararajan, S., 2017. Evidence-Based Trust Evaluation System for Cloud Services Using Fuzzy Logic. International Journal of Fuzzy Systems, 19(2), pp.329-337.

[30] Su, T.J., Wang, S.M., Vu, H.Q., Ku, D.Y. and Huang, J.L., 2016, July. An Application of Fuzzy Theory to the Power Monitoring System in Cloud Environments. In Computer, Consumer and Control (IS3C), 2016 International Symposium on (pp. 350-354). IEEE.

[31] Wang, S., Liu, Z., Sun, Q., Zou, H. and Yang, F., 2014. Towards an accurate evaluation of quality of cloud service in service-oriented cloud computing. Journal of Intelligent Manufacturing, 25(2), pp.283-291.

[32] Zavvar, M., Rezaei, M., Garavand, S. and Ramezani, F., 2016. Fuzzy Logic-Based Algorithm Resource Scheduling for Improving The Reliability of Cloud Computing. Asia-Pacific Journal of Information Technology and Multimedia, 5(1).

[33] Amini, A., Jamil, N., Ahmad, A.R. and Sulaiman, H., 2017, April. A Fuzzy Logic Based Risk Assessment Approach for Evaluating and Prioritizing Risks in Cloud Computing Environment. In International Conference of Reliable Information and Communication Technology (pp. 650-659). Springer, Cham.

[34] Subbulakshmi, K., 2014. Antilock-Braking System Using Fuzzy Logic. Middle-East Journal of Scientific Research, 20(10), pp.1306-1310.

[35] Mago, V.K., Bhatia, N., Bhatia, A. and Mago, A., 2012. Clinical decision support system for dental treatment. Journal of Computational Science, 3(5), pp.254-261. 